\title{
Cos and Sine (CAS) Pre-coding for PAPR drop in OFDM systems
}

\author{
Sadu Venkata Naresh, P. V. Naganjaneyulu, P. Nageswara Rao
}

\begin{abstract}
Extreme Peak to Average Power Ratio (PAPR) is as yet the common significant test in Orthogonal Frequency Division Multiplexing (OFDM) framework. A consolidated strategy of Discrete Cosine Transform (DCT) and Discrete Sine Transform (DST) precoding based CSD procedure for PAPR decrease in OFDM frameworks are represented in this article. Here, the constants are spoken to utilizing a canonic signed digit (CSD) depiction. For bit-positions relating to a positive digit in the CSD portrayal, the bit-item is included that segment. For negative digits in the CSD portrayal, a one-piece two's supplement portrayal is utilized. To evade sign-expansion a remuneration vector is utilized. Analyzed results demonstrate that our proposed procedure can diminish the PAPR performance very effectively than following the conventional PAPR lessening methods.
\end{abstract}

Index Terms - DCT \& DST coding, CSD, Companding, Precoding

\section{INTRODUCTION}

OFDM framework has been utilized for the fast speed computerized interchanges which incorporates Digital audio \& video broadcasting, High-definition TV and ADSL (Asymmetric Digital Subscriber Line) because of vigor to the thin band impedance and extraordinary wide-course blurring [1]. The most significant detriment of OFDM stays the aforementioned high PAPR dispensing grave corruption in execution after non-linear power intensifier (PA) is utilized. This unnecessary PAPR powers the communicate PA to take an Input back off so as toward making certain direct strengthening of the sign, consistently lessens the effectiveness of the enhancer. Greater PAPR wants great goals for the collector analog to digital converter (A/D). As with the lively scope of the signal is significantly bigger aimed at, over the top PAPR, the peak goal quantizer is imperative to diminish quantization blunders, that calls for additional noteworthy bits and spots multifaceted nature then influence trouble at the collector front end. Numerous answers have kept on proposing to decrease PAPR which incorporates Selective Mapping (SLM), Coding techniques, Partial Transmit Sequences (PTS), Data permutation, etc[2]. The majority of those arrangements have limitations on machine parameters comprising of extensive assortment of sub-transporters, summary organization, and heavenly body type. Signal twisting

SaduVenkataNaresh, Research Scholar, JNTUH, Hyderabad, Telangana, India.(Email: svenkatanaresh@gmail.com)

Dr. P. V. Naganjaneyulu, Principal \& Professor of ECE, SMCE, Guntur, A.P, India.(Email: pvnaganjaneyulu@gmail.com)

Dr. P. NageswaraRao, Director \& Professor of ECE, MPES,Guntur, A.P, India.(Email: pnrao33@gmail.com)
Revised Version Manuscript Received on 10, September 2019.

arrangements along with cut-out [3][4][5] and companding can be utilized irrespective of the confinement over the gadget restrictions anyway by the cost of extended bit error rate and phantom regrowth. Despite the fact that cutting performs completely with low tweak orders, cutting blunder [8] will turn out to be enormous with higher requests and essentially degrades execution resulting in companding extra appropriate for high information rates applications. Later, Wang introduced the notable plan named $\mu$-law companding strategy (or named traditional companding, Wang plot) in view of discourse preparing, and it shows preferable in general execution over that of section approach [6]. In [7] Enchang Sun et. $\mathrm{Al}$ introduced a precoding process based on DCT for PAPR decrease in MSE-OFDM model and they guarantee by means of reproductions that DCT essentially based precoding approach can significantly lessen the PAPR without lifting the image mistakes.

We present another joined DCT and DST pre-coder aimed at PAPR decrease. The demonstrated paper utilizes DCT based pre-coder that stays considerably fewer mind-boggling than various precoders [9] after the increase of section turn issue and formerly the IFFT inside the SLM OFDM machine. CAS (Cos and Sine) pre-coder based absolutely CSD approach is signal autonomous and it doesn't require any entangled streamlining strategy has been proposed.

This paper is sorted out with the end goal that in segment II, portrays OFDM framework basics and PAPR decrease, the proposed model rendition for PAPR decrease and PC reproduction impacts in Section III and Section IV and segment V closes the paper.

\section{METHODOLOGY}

\section{OFDM System}

An OFDM framework parts the inordinate rapidity information stream into some of equivalent short information rate streams and they are communicated all the while over some of symmetrical sub-transporters. Fig. 1 displays the square chart of an OFDM model. The base-band tweaked images are gone over $\mathrm{S} / \mathrm{P}$ converter which produces a composite vector of length $\mathrm{N}$ as $\mathrm{X}=[\mathrm{X} 0, \mathrm{X} 1, \mathrm{X} 2 \ldots \mathrm{XN}-1]^{\mathrm{T}}$ and afterward $\mathrm{X}$ is then gone into the IFFT square. The composite baseband OFDM signal with $\mathrm{N}$ sub-carriers can be composed with

$$
\begin{aligned}
& x_{n}=\frac{1}{\sqrt{N}} \sum_{k=0}^{N-1} X_{k} e^{j 2 \pi} \frac{n}{N} k, n=0,1,2 \ldots N-1 \\
& \text { In which } \mathrm{j}=\sqrt{-1} \text { and OFDM's PAPR }
\end{aligned}
$$




$$
\boldsymbol{P A P R}=\frac{\max \left|x_{n}\right|^{2}}{E\left[\left|x_{n}\right|^{2}\right]}
$$

where E [.] indicates Expectation and the Complementary Cumulative Distribution Function (CCDF) of an OFDM signal can be composed with

$$
\boldsymbol{P}\left(\boldsymbol{P A P R}>\boldsymbol{P A P R} \boldsymbol{R}_{0}\right)=1-\left(1-e^{-P A P R o}\right)^{N}
$$

$P A P R_{0}$ is the cutting edge [10] besides the equivalence can remain recited as the

Figure 1. OFDM system

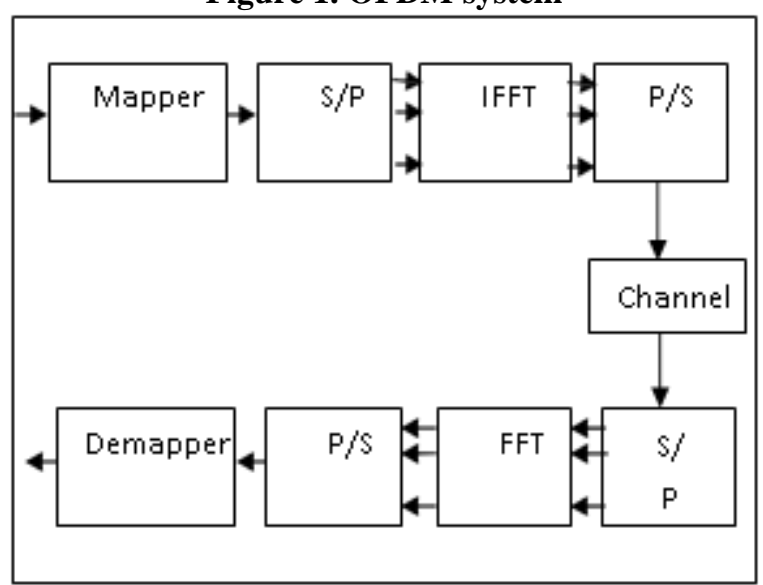

possibility that character block goes over some pin near $P A P R_{0}$.

\section{PRECODER BASED OFDM STRUCTURE}

In this area we present the precoding framework model basically dependent on

\section{The Discrete Cosine Transform (DCT) Technique}

OFDM system-based precoding applied block diagram is shown in Fig. 2 in which the system, a precoding network $P$ of measurement $\mathrm{N} \times \mathrm{N}$ is fabricated that depends on DCT. $\mathrm{P}$ is done to groups of stars images sooner than IFFT to diminish the power ratio. DCT framework $\mathrm{P}$ of length $\mathrm{N}-\mathrm{by}-\mathrm{N}$ might be made by method for the use of condition

$$
\boldsymbol{D}_{i j}=\left\{\begin{array}{rr}
\frac{1}{\sqrt{\bar{N}}}, & i=0,0 \leq j \leq N-1 \\
& \sqrt{\frac{2}{N}} \cos \frac{\pi(2 j+1) j}{2 N}, \mathbf{1} \leq \boldsymbol{i} \leq \boldsymbol{N}-\mathbf{1}, 0 \leq j \leq N-1
\end{array}\right.
$$

and DCT can be defined as

$$
X_{k}=\sum_{n=0}^{N-1} x_{n} \cdot \cos \left[\frac{\pi}{N}\left(n+\frac{1}{2}\right) k\right]
$$

in which $\mathrm{k}=0,1 \ldots, \mathrm{N}-1$. In precoding based OFDM framework, base band adjusted records are gone with $\mathrm{S} / \mathrm{P}$ convertor creating a confused matrix of size $\mathrm{N}$ composed of $\mathrm{X}=[\mathrm{X} 0, \mathrm{X} 1, \mathrm{X} 2 \ldots \mathrm{XN}-1]^{\mathrm{T}}$. At that point pre-coding is useful to this convoluted path, changes its mind-boggling vector into new one of period $\mathrm{N}$ composed of

$$
Y=P X=\left[Y_{0}, Y 1, Y 2_{\ldots} Y_{N-1}\right]^{T}
$$

\section{The Discrete Sine Transform (DST)Technique}

The Precoding is carried out formerly data input collection exceeded to the Inverse FFT for lowering auto-correlation of the information input series to decrease PAPR and the extent of the DST pre-coder is stored same as IFFT size the DST. For an input sequence $b_{n}$, the output of using DST may be defined as

$$
y_{n}=\sum_{k=0}^{N-1} b_{n} B_{n} \sin \left[\frac{n \pi t}{T_{s}}\right], k=1, \ldots N-1
$$

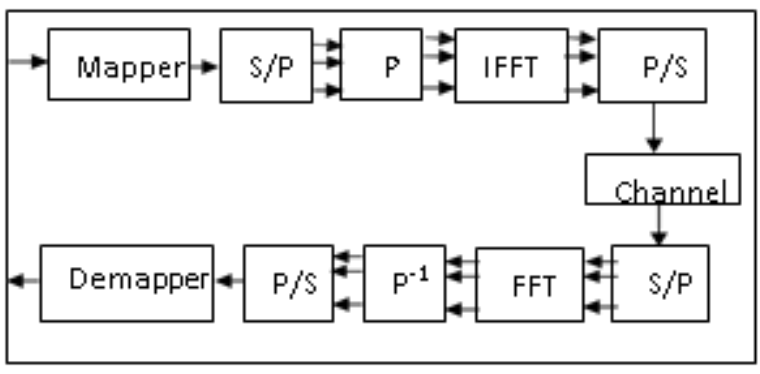

Figure 2. Block drawing of Precoding based OFDM system.

in which $b_{n}$ is the contribution to the Precoding transform, $\mathrm{y}_{\mathrm{n}}$ is creation of the pre-coding remodel and $\mathrm{Bn}$ is the coefficient of the Discrete Sine Transform technique that's expressed as

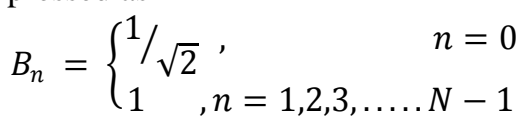

\section{CSD Multiplier}

The Canonic signed digit (CSD) representation is used to realize the function of complicated multiplier in non-trivial multiplication. The real and imaginary elements of the consistent element are represented in CSD form. The corresponding elements of the non-constant thing are represented in two's supplement shape.

\begin{tabular}{|l|l|l|}
\hline $\begin{array}{l}\text { Coefficient } \\
\text { Value }\end{array}$ & 16 Bit Expression \\
\cline { 2 - 3 } & Binary & CSD \\
\hline 0.923828 & 0111011001000000 & $100 \overline{1} 10 \overline{1}$ \\
& & 001000000 \\
\hline 0.707092 & 0101101010000010 & $0110 \overline{1}$ \\
& & 01010000010 \\
\hline 0.382629 & 0011000100000110 & $010 \overline{1} 0001000010$ \\
& & $\overline{1}$ \\
\hline
\end{tabular}

Table 1. Representation of binary and CSD

This approach has capacity benefit of simpler circuit systems than the traditional complex multiplier. The constants are indicated by means of a canonic signed-digit (CSD) illustration. For bit-positions similar to a high-quality digit inside CSD illustration the bit multiplication is supplementary in that column. For negative digits within the CSD representation, a one-bit two's supplement illustration is used. To keep away from signal delay a return vector is used. The CSD unit includes many shifters and adders. It is used to understand the multiplication whilst the one factor is constant. The CSD unit is used to assemble quantity that carries the minimal possible number of non-0 bits.

\section{SIMULATION RESULTS}

Simulations in MATLAB have been done for accessing the performance of described method. 

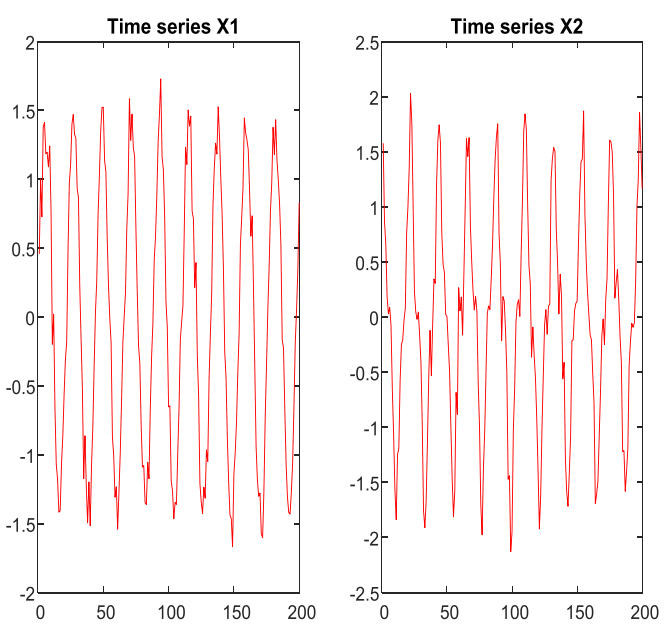

Fig.3. Time Series

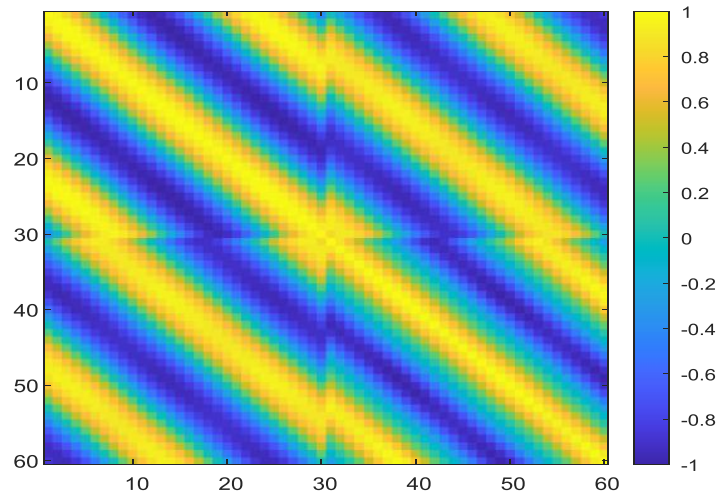

Fig.4. Co-variance matrix
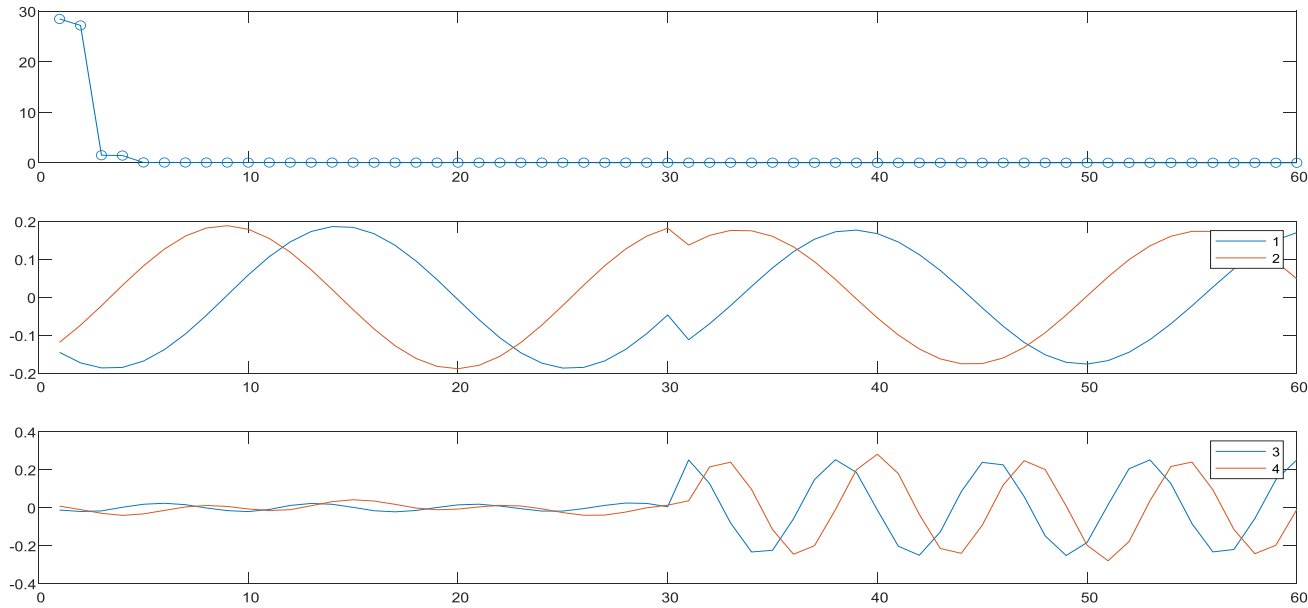

Fig.5. Cos \& Sine Pre-coding for PAPR reduction
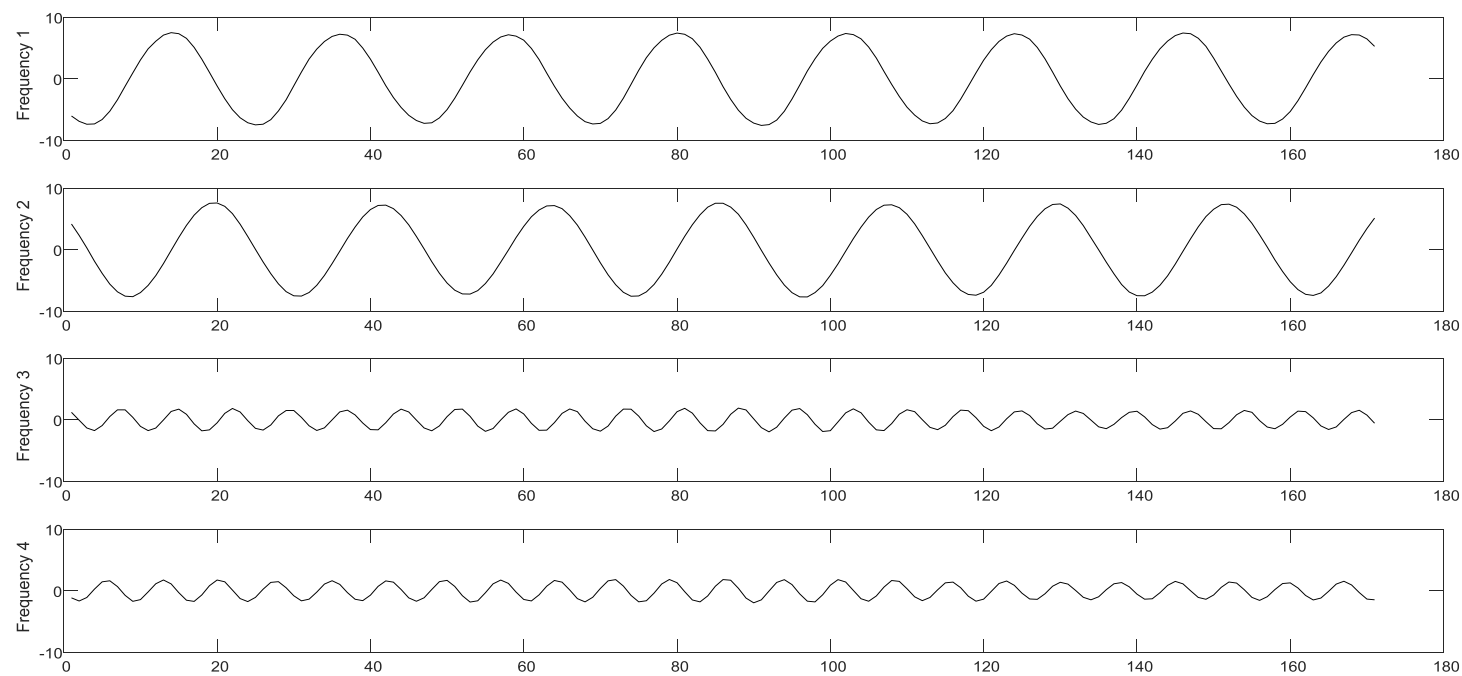

Fig.6. Reducing the PAPR

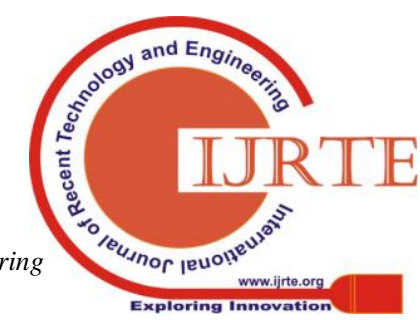



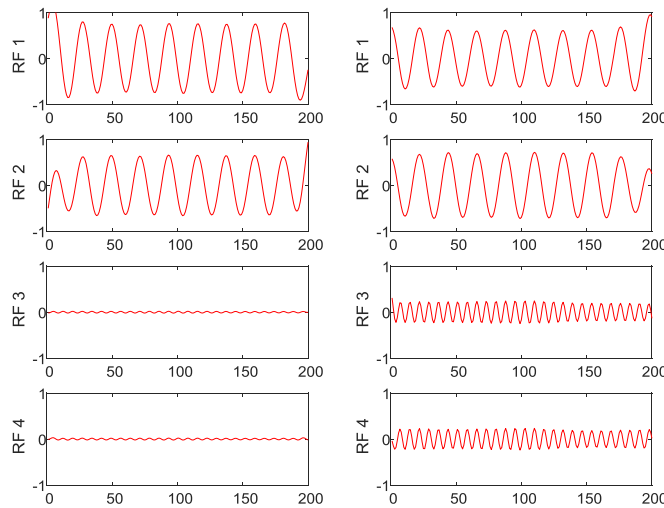

Fig.7. OFDM Signal
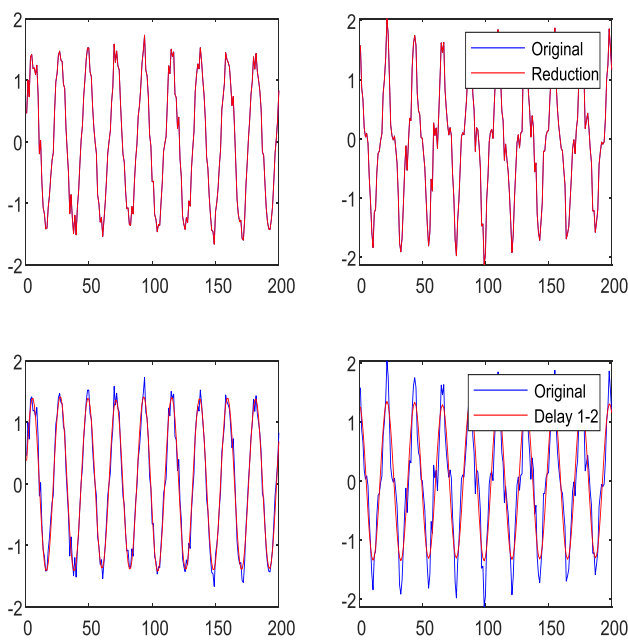

Fig.8. RF High Signals

\section{V.CONCLUSION}

In this paper, we recommend a better-quality joint pre-coding technique dependent on two precoding plans (DCT and DST), here called as Cos \& Sine (CAS) Pre-coding method shows better simulation results when compared with the previous techniques used. With the usage CSD that uses bits converted from binary to canonic signed digits.

\section{REFERENCES}

1 . Hikmet, K. Georges, and J.Jsabelle, "Transmission techniques for digital terrestrial TV broadcasting," IEEE Comm. Magazine, pp. 100-109, Feb.1995.

2 C. Schurgers and M. Srivastava, "A systematic technique to top-to-average strength ratio in OFDM," in Proc. SPIE, 2001, vol. 4474, pp. 454-464.

3 J. Armstrong, "Peak-to-average reduction for OFDM by using repeated clip- ping and frequency domain filtering," IEE Electron. Lett., vol. 38, pp. 246-247, May 2002.

$4 \mathrm{X}$. Li and L. J. Cimini, Jr., "Effects of clipping and filtering at the in step with- formance of OFDM," IEEE Commun. Lett., vol. 2, no. 5, pp. 131-133, May 1998.

5 S. C. Thompson, J. G. Proakis, and J. R. Zeidler, "The effectiveness of signal clipping for PAPR and total degradation reduction inOFDM systems," in Proc. of the IEEE Global Telecommun. Conf., St. Louis, Dec. 2005, vol. 5, pp.2807-2811.

6 X. Wang, T. T. Tjhung, and C. S. Ng, "Reduction of peak-to-average power ratio of OFDM system using a companding technique," IEEE Trans. Broadcast., vol. 45 , no. 3, pp. 303-307, Sept.1999.

7 Enchang Sun, Kechu Yi, Bin Tian and Wang, X., "A method for PAPR reduction in MSE-OFDM systems," Advanced Information Networking and Applications, 2006. AINA 2006. 20th International Conference on vol.2, no., pp. 4 pp.-, 18-20 April2006

8 Sohn I, Kim S C. "Neural network based simplified clipping and filtering technique for PAPR reduction of OFDM signals," IEEE Communications Letters, vol. 19, no. 8, pp. 1438-1441, Aug. 2015.

9 S. Hee Han and J. Hong Lee, "An overview of top-to-common strength ratio reduction strategies for multicarrier transmission", IEEE Trans. Wireless Communications, Vol.12, Iss.2, April 2005, Pages: 56sixty five

10 Luqing Wang and ChinthaTellambura,"A Simplified Clipping and Filtering Technique for PAR Reduction in OFDM Systems", Signal Processing Letters, IEEE , vol.12, no.6, pp. 453-456, June 2005. 